\title{
Forward-looking infrared imaging predicts ultimate burn depth in a porcine vertical injury progression model
}

\author{
Joseph Miccio ${ }^{1}$, Shruti Parikh², Xavier Marinaro ${ }^{5}$, Atulya Prasad ${ }^{2}$, Steven McClain ${ }^{3}$, \\ Adam J Singer ${ }^{3}$, and Richard AF Clark ${ }^{2,4}$
}

\footnotetext{
${ }^{1}$ School of Medicine, ${ }^{2}$ Department of Biomedical Engineering, ${ }^{3}$ Department of Emergency Medicine, ${ }^{4}$ Department of Dermatology, Stony Brook University, Stony Brook, NY 11794; ${ }^{5}$ Biological Engineering, Cornell University, Ithaca, NY 14853
}

\section{Contact Information}

\section{Richard AF Clark, MD}

Departments of Biomedical Engineering and Dermatology

HSC T-16, 060

Stony Brook University

Stony Brook, NY 11794-8165

richard.clark@stonybrook.edu 


\begin{abstract}
Introduction

Current methods of assessing burn depth are limited and are primarily based on visual assessments by burn surgeons. This technique has been shown to have only $60 \%$ accuracy and a more accurate, simple, noninvasive method is needed to determine burn wound depth. Forward-looking Infrared (FLIR) thermography is both noninvasive and user-friendly with the potential to rapidly assess burn depth. The purpose of this paper is to determine if early changes in burn temperature (first 3 days) can be a predictor of burn depth as assessed by vertical scarring 28 days post-injury.
\end{abstract}

\title{
Methods
}

While under general anesthesia, 20 burns were created on the backs of two female Yorkshire swine using a 2.5 cm x $2.5 \mathrm{~cm}$ x $7.5 \mathrm{~cm}, 150$-gram aluminum bar, for a total of 40 burns. FLIR imaging was performed at both early (1, 2 and 3 days) and late (7, 10, 14, 17, 21, 24 and 28 days) time points. Burns were imaged from a height of 12 inches from the skin surface. FLIR ExaminIR @ software was used to examine the infrared thermographs. One hundred temperature points from burn edge to edge across the center of the burn were collected for each burn at all time points and were exported as a comma-separated values (CSV) file. The CSV file was processed and analyzed using a MATLAB program. The temperature profiles through the center of the burns generated parabola-like curves. The lowest temperature (temperature minimum) and a line midway between the temperature minimum and ambient skin temperature at the burn edges was defined and the area of the curve calculated (the “temperature half-area”).

\section{Results}

Half-area values 2 days post-burn had higher correlations with scar depth than did the minimum temperatures. However, burns that became warmer from 1 day to 2 days after injury had a lower scar depth then burns that became cooler and this trend was best predicted by temperature minima. When data were analyzed as a diagnostic test for sensitivity and specificity using >3mm scarring, i.e. a full-thickness burn, as a clinically 
relevant criterion standard, temperature minima at 2 days post-burn was found to be the most sensitive and specific test.

\section{Conclusions}

FLIR imaging is a fast and simple tool that has been shown to predict burn wound outcome in a porcine vertical injury progression model. Data showed that more severe burn wounds get cooler between 1 and 2 days postburn. We found four analytic methods of FLIR images that were predictive of burn injury progression at 1 and 2 days post-burn; however, temperature minima 2 days post-burn appeared to be the best predictive test for injury progression to a full-thickness burn. Although these results must be validated in clinical studies, FLIR imaging has the potential to aid clinicians in assessing burn severity and thereby assisting in burn wound management.

Keywords

Forward looking infrared, thermography, burn, porcine model, diagnostic 


\section{INTRODUCTION}

Therapy used for burn injury patients depends on the depth of the burn. Superficial dermal burns heal spontaneously within three weeks and require minimal medical intervention. However, deep dermal burns, as well as full thickness burns, require excision and grafting and can take many months to heal. Furthermore, earlier excision and grafting lead to better patient outcomes, including faster healing, lower incidence of infection, and reduced length of hospital stay [1, 2]. Thus, physicians must be able to rapidly assess burn depth in order to provide optimal burn care.

Unfortunately, current methods of assessing burn depth are limited. Most burn surgeons rely on a visual assessment, which has been shown to have only $60 \%$ accuracy, even in the eyes of the most experienced surgeons [3]. In light of this knowledge, clinical investigators have evaluated the use of alternate ways to assess burn severity. Laser Doppler Imaging (LDI), which can give an indirect qualitative measurement of blood flow through the dermis, has been shown to increase the accuracy of burn depth prediction at time points as early as 3 days [4]. However, LDI is cumbersome and costly and requires the patient to remain still for up to 30 minutes, which is impractical in situations where patients are experiencing a significant amount of pain. Furthermore, LDI is not accurate during the first 1-2 days after injury. Hence, this technology is not widely used outside of research studies. Indocyanine green angiography is an alternative method for burn assessment. This technology is invasive and costly, and therefore, is not a realistic choice for assessment of burn depth in the clinic. It may find use, however, during surgical debridement in order to reduce the margins of viable tissue that are removed $[2,5]$

Forward looking infrared (FLIR) thermography has the potential to rapidly assess burn depth in a noninvasive, quantitative fashion. The technology measures tissue thermal emission as radiation in the far IR

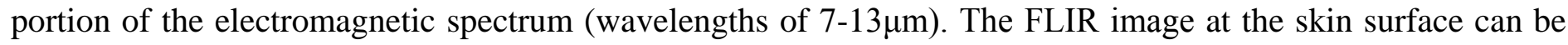
viewed as the integral of temperature throughout the layers of the skin, since the camera captures thermal emission from tissue down to a depth of $3 \mathrm{~cm}$. Since deep burns have more blood vessel injury, there will be less tissue perfusion and thus less thermal emission up through the skin [6]. The metabolic rate of cells damaged by thermal injury is also reduced resulting in less emission of heat. 
Several groups have reported that thermography has the potential to improve clinical assessment of burn depths [7-9]. These previous studies focused more on exploring the use of thermography in a small set of patients. Each of these groups used a single temperature point in the burn in order to make their judgment. Using more than one temperature point, however, may lead to a better prediction of burn depth. Additionally, these studies used one time point in determining burn wound outcome. Knowing the change in burn wound temperature in the period of time following injury may provide potentially valuable information about the outcome of the burn injury. The purpose of this study is to determine if the change in burn temperature during the first 3 days after injury can accurately predict burn depth as assessed by vertical scarring 28 days post injury. In addition, we also assessed if the use of multiple temperature points better predicts vertical scarring than a single temperature point. The study also serves to standardize the implementation and interpretation of FLIR thermography as a precedent to a clinical trial.

\section{Methods AND MATERIALS}

\subsection{Study Design}

Investigators blinded to injury progression outcome performed burn analysis using FLIR data in a porcine vertical burn injury progress model [10]. Our study protocol was conducted following Institutional Animal Care and Use Committee (IACUC) Research Review Board approval.

\subsection{Animal Use}

This study utilized two female Yorkshire swine (20-25 kg). Animals were allowed to acclimate for at least one week during which they were given a standard diet until they were fasted overnight prior to experimental procedures. Appropriate housing and care for animals was ensured in our Division of Laboratory Animal Resources (DLAR) in accordance with National Research Council Guidelines [11]. In the animal quarters the ambient temperature is $71.36 \pm 0.59 \mathrm{~F}$ and humidity is $56.82 \pm 2.83 \% \mathrm{rh}$.

\subsection{Burn Creation and Management}


Animal sedation and intubation were performed by the methodology previously described by Singer et al. [10]. While under general anesthesia, 20 burns were created on the back of each animal using a $2.5 \mathrm{~cm} \times 2.5 \mathrm{~cm}$ x 7.5 cm, 150-gram aluminum bar (Small Part Inc, Miami Lakes, FL) heated in a water bath at $70^{\circ} \mathrm{C}$ or $80^{\circ} \mathrm{C}$. The block was then promptly blotted dry, so as to not cause burn injury from steam or scalding, then applied perpendicular to the skin, with $2 \mathrm{~kg}$ of pressure for a period of 20 or 30 seconds. The nomenclature used in this paper when describing burns is temperature/time (e.g. $70^{\circ} \mathrm{C}$ bar held to the animal for 30 seconds is denoted as a 70/30 burn).

Immediately following burns, the burn perimeter, site number, and study identification number were tattooed on the animals using an electric tattoo animal marker (Huck Spaulding Enterprises, New York, USA). The tattooed areas were then gently washed with mild soap and water and patted dry. Following the tattooing procedure, the necrotic epidermis was removed from the burns by gently scraping with the handle of a forceps or with gauze in order to simulate burn blister rupture in humans. Photographs were taken with a digital camera with a ruler for scale. Full thickness $3 \mathrm{~mm}$ punch biopsies were taken at 1, 2, 3, 7 days post-burn and $6 \mathrm{~mm}$ punch biopsies were taken at 28 days post-burn. Euthanasia was performed 28 days post-burn using pentobarbital.

\subsection{FLIR Imaging}

FLIR images were acquired using a FLIR T300 camera (FLIR Systems, Inc, Wilsonville, OR) at early (1, 2 and 3 days) and late (7, 10, 14, 17, 21, 24, and 28 days) time periods after injury. Burns were imaged in groups of four and the image was taken from a distance of 12 inches, measured by a ruler, oriented perpendicular to the skin. 10 minutes before imaging, the wounds were cleansed with a soap water solution and air-dried. It was of great importance that the wounds are dry, in order to prevent a falsely low temperature due to evaporative heat loss [12]. In addition, the overhead operating room light was moved at least 3 feet above the skin surface and at this height was shown to have no impact on skin temperature. Any biopsies scheduled for the same day were taken after FLIR imaging was completed. Biopsies at 1, 2, 3 and 7 days post-burn were taken at the corners of the wound and were found to have no influence on temperature across the center of the wound. FLIR imaging 
was performed at the following time points: 1 day, 2 days, 3 days, 7 days, 10 days, 14 days, 17 days, 21 days, 24 days, and 28 days.

\section{$2.5 \quad$ Histopathological assessments}

Day 28 biopsies were used to determine scar depth. All biopsies were alcohol-dehydrated, xylene-cleared, and paraffin embedded. The biopsies were bisected, sectioned at 5mm intervals, and stained with Hematoxylin \& Eosin. A board certified dermatopathologist, masked to burn parameters, evaluated all tissue sections using standard light microscopy. For each half of a bisected biopsy, three vertical scar depth measurements, evenly spaced across the wound specimen, from the dermal-epidermal junction to the base of the scar were acquired for a total of 6 measurements per biopsy (Figure 1). The average of the 6 scar depth values for each burn was used in analysis.

\subsection{Data Analysis}

LIR ExaminIR@ software was used to examine infrared thermographs. A horizontal line was drawn through the middle of the burn halfway between the upper and lower edge of the burn (Figure 2A). Each pixel along the line is associated with the temperature at that location (Figure 2B). The 100-pixel (temperature) line of data was exported as a comma-separated value (CSV) file into a MATLAB software program (Mathworks, Natick, MA). Once the temperature minima and ambient skin temperatures at the burn edges were defined, the area between the temperature minima and a line midway between the temperature minima and the ambient skin temperature at the burn edges ("temperature half-area”) was calculated (Figure 2B). This measurement was chosen since it represents the summation of temperatures across the burn.

Different parameters of the temperature profile (temperature minima, temperature half-areas, change in temperature minima and temperature half-area) at different time points were then analyzed for correlation with scar depth in Microsoft Excel. Receiver operator characteristic (ROC) curves were also performed in Excel to determine optimal sensitivity and specificity for each of the parameters of the FLIR images as no predetermined gold standard was available. Since the variance of burn temperatures and scar depth were greater within an animal than between animals, the data from the two pigs were pooled. 


\section{Results}

First we assessed whether the absolute temperature minima or the temperature half-area at any single time point would be predictive of scar depth at 28 days (Table 1). Although the most superficial burns (70/20) had the lowest average temperature minima at 1 day among all burn types, these data were not statistically significant. Furthermore, while the correlation coefficient of temperature minima at 1 day showed a slightly positive correlation between increased temperature and burn progression, the coefficient of determination $\left(\mathrm{R}^{2}\right)$ was only 0.188 indicating no statistical correlation (Figure 3A), confirming that temperature minima at 1 day post-burn was an unreliable tool for predicting ultimate burn depth. Temperature half-area of the 70/20 burns at 1 day showed the smallest value of all burn types (Table 1), which was consistent with these burns having the least scar depth; however, the $\mathrm{R}^{2}$ was only 0.22 (Figure 4A) demonstrating that this measure was also unreliable at predicting injury progression at 1 day post-burn.

Temperature minima at 2 days post-burn showed modest correlation with scar depth (Figure 3B), however, temperature minima at 3 and 7 days showed poor correlations (Figure 3C, D). In contrast, temperature half-area at 2, 3 and 7 days post-burn showed appreciably better correlation with scar depth compared to the temperature minima at each time point, respectively (Figure 4B, C, D). Of these the 2-day time point for temperature half-area had the strongest correlation with scar depth, which was consistent with temperature minima data over the same time periods (Figure 3B). Time points later than 7 days showed poor correlation with 28d scar depth, and were thus not included in this report.

Next we evaluated whether the change in temperature minima or temperature half-area between 1 and 2 days, or between 2 and 3 days would be predictive of future scar depth (Table 2). Both change in temperature minima and change in temperature half-area from day 1 to day 2 predicted that burns that became cooler had a deeper scar depth, and burns that became warmer had a more superficial scar depth. A confounding finding was that burns that became warmer from 2 to 3 days (decreased temperature half-area or increased minima) had an increased scar depth on average. $\quad$ Results, which are shown as averages in Table 2, are depicted visually in Figures 5 and $\mathbf{6}$ as scatter diagrams. Of these, clearly the temperature minima change between day 1 and day 2 correlated best with increased scar depth at day 28 (Figure 5a) 
Although the linear correlations presented in this study were modest, the data may hold potential to be used clinically. Thus, all data in Figures 3-6 were analyzed as diagnostic tests for scar depth, and the best sensitivity and specificity was found when analyzing Figure 3B, Figure 4B, Figure 5A, and Figure 6A. Since no gold standard exists for predicting the depth of a burn scar, receiver operator characteristic (ROC) curves were used to determine optimal sensitivity and specificity of the different FLIR modalities for scar at different depths. The plots for each of these tests are shown in Figure 7.

When calculating sensitivity and specificity, a true positive value is defined based upon the diagnostic test in question. In this study true positive was defined as a temperature minima that was less than or equal to a value that predicted a scar depth greater than a specified cutoff. For half-area measurements, a true positive was defined as a half-area greater (lower temperature) than or equal to a value that predicted a scar depth greater than a specified cutoff. Thus, for each generated ROC curve the scar depth was set as an arbitrary constant and the temperature minima or half-area cutoff value was varied. These analyses were performed at arbitrary scar depths of $1,2,3,4$ or $5 \mathrm{~mm}$ resulting in a series of curves for each diagnostic tests. The scar depth and temperature minima/temperature half-area values used that led to the best sensitivity and specificity for each diagnostic test are displayed in Table 3.

In our porcine model a scar depth greater than 3mm was considered full-thickness burns since the interface of the dermis and subcutaneous layer was approximately $3 \mathrm{~mm}$. Since it is clinically relevant to determine if a burn wound will progress to a full-thickness injury, a 3mm scar in this study was of particular interest. Of the four diagnostic tests, temperature minima at day 2 and temperature half-area at day 2 produced optimal sensitivity and specificity at a scar depth cutoff of $\geq 3 \mathrm{~mm}$.

\section{Discussion}

The data presented in this paper provide preliminary results supporting the future use of FLIR imaging in the clinical setting to assess burn injuries. When making predictions of scar depth with a single FLIR image at 2 days post-burn, both temperature minima and an increased temperature half-area, which indicates lower

temperature across the burn (Figure 2), correlated with scar depth $\left(\mathrm{R}^{2}=0.5959\right.$ and 0.6713 , respectively). 

over the first 48 hours post-burn (Clark et al., unpublished observations), which would likely lead to less heat generation secondary to poor perfusion and decrease stromal cell metabolism.

When FLIR image analyses were evaluated as diagnostic tests, the best sensitivity and specificity was achieved with temperature minima at day 2 as a predictor of scar depth $\geq 3 \mathrm{~mm}$. Importantly, 3mm was the average distance between the dermal-epidermal junction and dermal-subcutaneous interface in the animals used for this study, thus the temperature minima at day 2 was a predictor of full thickness burn. Although one might expect data giving the strongest correlation coefficient would also constitute a better clinical test, this was not observed.

When measuring trends from 1 to 2 days, the change in temperature minima to predict burn depth proved superior to the change in half-area. An appropriate question to ask is why is the trend of a single point more accurate than the trend of an integral of multiple temperature points? This may be due to the logistics of data collection and analysis. The exact minimum temperature value was found by the ExaminIR@ software, while the line through the burn thermographic midpoint was drawn by an investigator. Furthermore, it was difficult, if not impossible, to draw a line through the same exact points on a subsequent day. Thus, each halfarea taken from the same burn at different time points could have been from a different line of pixels.

All four modalities of analyzing the FLIR images led to diagnostic tests with reasonable test characteristics. Although the use of change in temperature minima and temperature half-area from day 1 to day 2 were not the best diagnostic tests in terms of sensitivity and specificity, they may actually hold the most clinical utility. Due to the comparative nature of these tests, many confounding variables influencing skin temperature will be cancelled out, including differences in ambient temperature and humidity between institutions and baseline skin temperature between patients. It would be reasonable to expect that a burn patient would be able to have imaging conducted at 1 day and 2 days after the burn wound. Thus, if these test parameters described in Table 3 are comparable to the values in humans, FLIR may have a role in the diagnosis of full thickness burns. A pilot clinical trial from our Institution suggests that this may be the case. 
When assessing trends from 2 to 3 days, the burns that become warmer actually have an increased scar depth, as described in Table 2. Consistent with this finding is the fact that there was a lesser association between temperature half-area and scar depth at 3 days compared to 2 days, as shown in Figure 3 . These findings may be due to an increasing amount of inflammation, and thus local heat transfer, associated with deeper burns by day 3. There have been no studies to date that have explored the local or systemic inflammatory response at early times post-burn in pig models. Thus, it is difficult to prove at this point that the increase in local inflammation by 3 days is significant enough to cause a reversal in the association found between FLIR imaging and scar depth from 1 to 2 days. However, it has been documented in rat models and in humans that the levels of many systemic proinflammatory cytokines increase between days 1 and 3 following a dermal burn injury [14-19]. A further study involving the histopathology of pig burn wounds at early time points is necessary to support the hypothesis that an increase in inflammation by day 3 in deep burns is the cause of the trend reversal in FLIR imaging vs. scar depth.

The following limitations must be considered. The results are retrospective and were acquired using FLIR images from previous studies. The sample size was small and consisted of 2 pigs for a total of 40 burns. Also, all wounds were square in shape, which is vastly different from the various burn wounds that present clinically. The current study used a standardized burn model in which the burns are very uniform. In contrast, many burns in humans are of mixed depth and it is unclear how FLIR would perform in these types of burns.

\section{Conclusions}

Determination of ultimate burn depth is a clinical problem that has not yet been solved in a practical way. The data presented in this paper show the potential for FLIR imaging to have a role in the determination of ultimate burn severity. With FLIR imaging of the burn at 1 and 2 days after injury, clinicians may be able to gauge trends in burn temperature, and use this information to assist in clinical management. Replication of the results in humans and remodeling of the analytical performance of the FLIR technology to permit differing burn geometries should be considered before FLIR can achieve maximal clinical utility. 


\section{FUNDING}

Funded by U.S. Army Medical Research and Materiel Command (USAMRMC), the Office of Naval Research (ONR), the Veterans Administration (VA) and the National Institute of Health (NIH). This award is supported by contract number W81XWH-08-2-0034.

\section{CONFLICT OF INTEREST}

The authors of this study have no conflicts of interest to declare. 


\section{$\underline{\text { References }}$}

[1] Sterling, J.P., D.M. Heimback, and N.S. Gibran, Management of the Burn Wound. ACS Surgery: Principles and Practice, 2010.

[2] Heimbach, D., et al., Burn depth: a review. World J Surg, 1992. 16(1): p. 10-5.

[3] Monstrey, S., et al., Assessment of burn depth and burn wound healing potential. Burns, 2008. 34(6): p. 761-9.

[4] Hoeksema, H., et al., Accuracy of early burn depth assessment by laser Doppler imaging on different days post burn. Burns, 2009. 35(1): p. 36-45.

[5] Fourman, M.S., et al., Indocyanine green dye angiography accurately predicts survival in the zone of ischemia in a burn comb model. Burns, 2014. 40(5): p. 940-6.

[6] Devgan, L., et al., Modalities for the assessment of burn wound depth. J Burns Wounds, 2006. 5: p. e2.

[7] Hardwicke, J., et al., A pilot evaluation study of high resolution digital thermal imaging in the assessment of burn depth. Burns, 2013. 39(1): p. 76-81.

[8] Liddington, M.I. and P.G. Shakespeare, Timing of the thermographic assessment of burns. Burns, 1996. 22(1): p. 26-8.

[9] Medina-Preciado, J.D., et al., Noninvasive determination of burn depth in children by digital infrared thermal imaging. J Biomed Opt, 2013. 18(6): p. 061204.

[10] Singer, A.J., et al., Validation of a vertical progression porcine burn model. J Burn Care Res, 2011. 32(6): p. 638-46.

[11] (U.S.), N.R.C., Guide for the care and use of laboratory animals, I.f.L.A.R.U.S. Committtee for the Update of the Guide for the Care and Use of Laboratory Animals, Editor. 2011, National Academies Press: Washington, DC. p. 220.

[12] Cole, R.P., et al., Thermographic assessment of burns using a nonpermeable membrane as wound covering. Burns, 1991. 17(2): p. 117-22.

[13] Hirth, D., et al., Endothelial necrosis at 1 hour postburn predicts progression of tissue injury. Wound Repair Regen, 2013. 21(4): p. 563-70.

[14] Tyler, M.P., et al., Dermal cellular inflammation in burns. an insight into the function of dermal microvascular anatomy. Burns, 2001. 27(5): p. 433-8.

[15] Faunce, D.E., et al., Neutrophil chemokine production in the skin following scald injury. Burns, 1999. 25(5): p. 403-10.

[16] Kataranovski, M., Z. Magic, and N. Pejnovic, Early inflammatory cytokine and acute phase protein response under the stress of thermal injury in rats. Physiol Res, 1999. 48(6): p. 473-82.

[17] Schwacha, M.G., et al., Impact of thermal injury on wound infiltration and the dermal inflammatory response. J Surg Res, 2010. 158(1): p. 112-20.

[18] Finnerty, C.C., et al., Cytokine expression profile over time in severely burned pediatric patients. Shock, 2006. 26(1): p. 13-9.

[19] Dehne, M.G., et al., Alterations of acute phase reaction and cytokine production in patients following severe burn injury. Burns, 2002. 28(6): p. 535-42. 
Figure 1: Deep dermal scar 28 days post burn. Blue lines indicate the linear orthogonal distance from the dermal-epidermal junction to the interface between the scar and underlying dermis. Measurements of the length of six such lines, three from each side of a bisected 28 day wound, were used to calculate the mean and standard deviation of a wound.

Figure 2. Calculation of burn "temperature half-area." A - Burn example as seen in FLIR ExaminIR@ software. A 100-pixel line was drawn horizontally across the burn of interest. B - The 100 temperature points were then exported as a CSV file and into a Matlab code that calculated temperature half-area.

Figure 3: Correlation of temperature minima with scar depth. A - Temperature minima at 1 day versus $S c a r$ depth $(P=.013)$. B - Temperature minima at 2 days versus scar depth $(P<.0001)$. $\mathbf{C}-$ Temperature minima at 3 days versus scar depth $(P<.053)$. D - Temperature minima at 7 days versus scar depth $(P<.0001)$.

Figure 4: Correlation of temperature half area with scar depth. A - Temperature half area at 1 day versus $S c a r$ depth $(P=.001)$. B - Temperature half area at 2 days versus scar depth $(P<.0001)$. C - Temperature half area at 3 days versus scar depth $(P$ $<.0001)$. $\mathrm{D}$ - Temperature half area at 7 days versus scar depth $(P<.0001)$.

Figure 5: A - Scar depth versus change in temperature minima from 1 to 2 days. If temperature decreases, most scars are over $3 \mathrm{~mm}$ deep whereas if temperature increases, most scars are less than $3 \mathrm{~mm}$ deep. B - Scar depth versus change in temperature minima from 2 to 3 days. The opposite is true - most burns where the temperature increased had a scar depth greater than $3 \mathrm{~mm}$, and most burns where the temperature decreased had a scar depth less than $3 \mathrm{~mm}$.

Figure 6: A - Scar depth versus change in temperature half area from 1 to 2 days. Overall, if area increases, most scars are $3 \mathrm{~mm}$ or greater. If area decreases, most scars are less than $3 \mathrm{~mm}$. B - Scar depth versus change in half area from 2 to 2 days. There is no discernable correlation here.

Figure 7: Diagnostic Test Parameters. A - Temperature minima at Day 2. With cutoff values of scar depth $\geq 3 \mathrm{~mm}$ and temperature minima at day $2 \leq 31.5^{\circ} \mathrm{C}$, the test achieved a sensitivity of $96 \%$ and a specificity of $100 \%$. B - Temperature Minima 1-2 Days. With cutoff values of scar depth $\geq 2 \mathrm{~mm}$ and change in minimum temperature $<1.7^{\circ} \mathrm{C}$ from day 1 to day 2 , the test achieved a sensitivity of $90 \%$ and a specificity of $89 \%$. C - Temperature half area at day 2 . With cutoff values of scar depth $\geq 3 \mathrm{~mm}$ and half-area of $>85$, the test achieved a sensitivity of $92 \%$ and a specificity of $93 \%$. D - Temperature half area $1-2$ days. With cutoff values of scar depth $\geq 2 \mathrm{~mm}$ and change in temperature half area $<-10$, the test achieved a sensitivity of $77 \%$ and specificity of $89 \%$. 


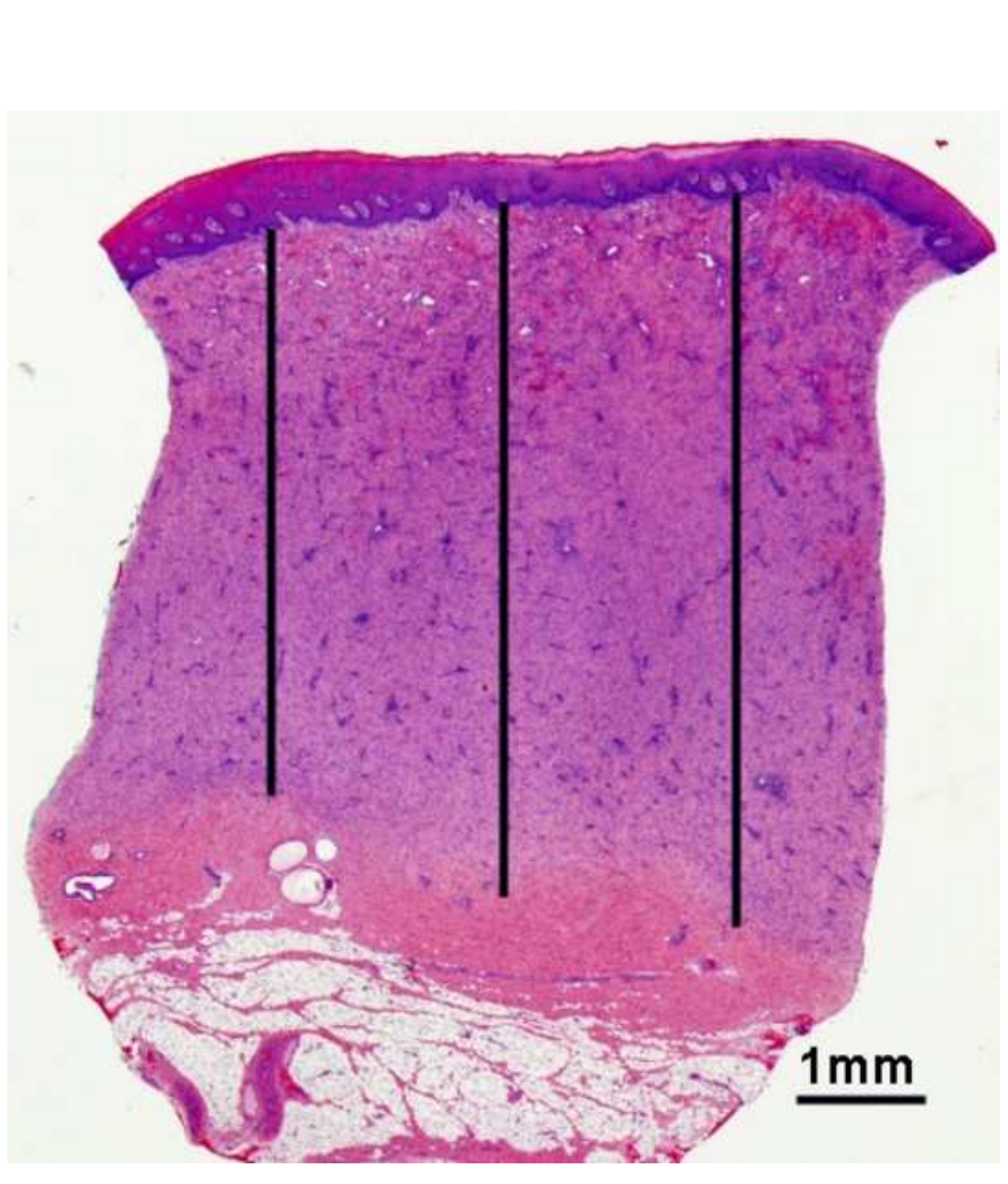

Figure 1

\section{Figure 1}

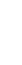

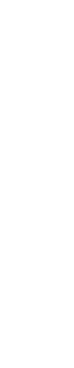



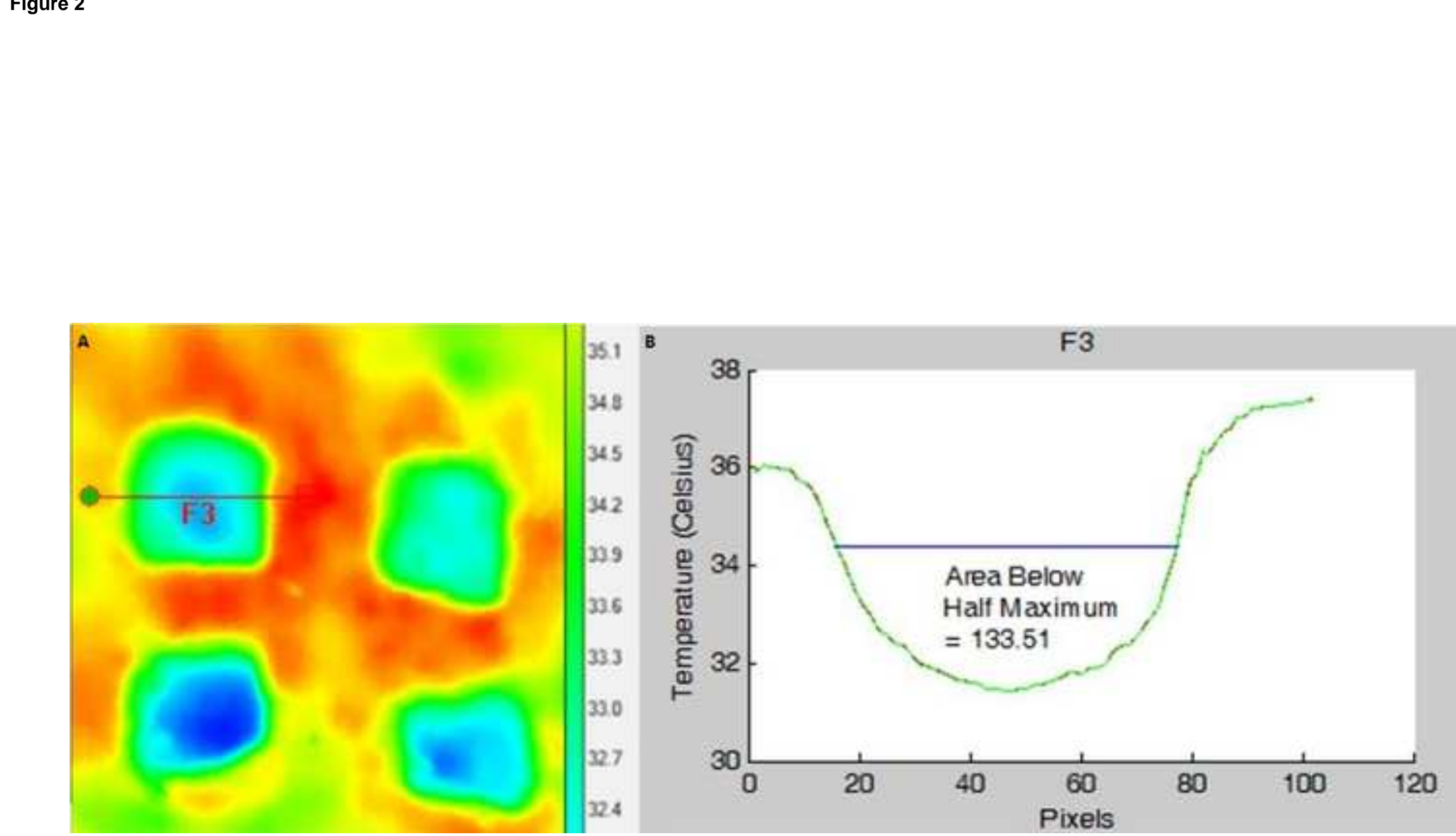


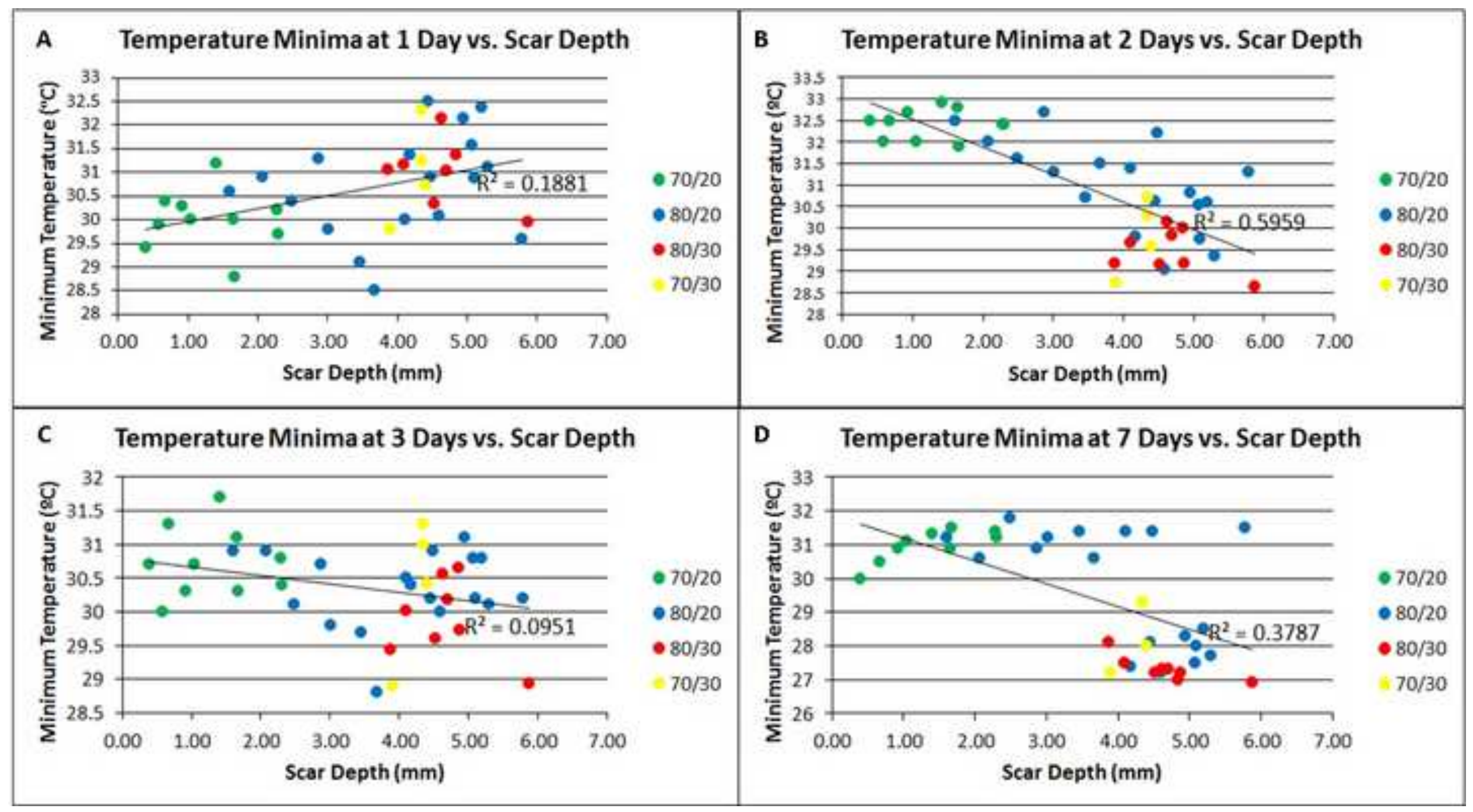




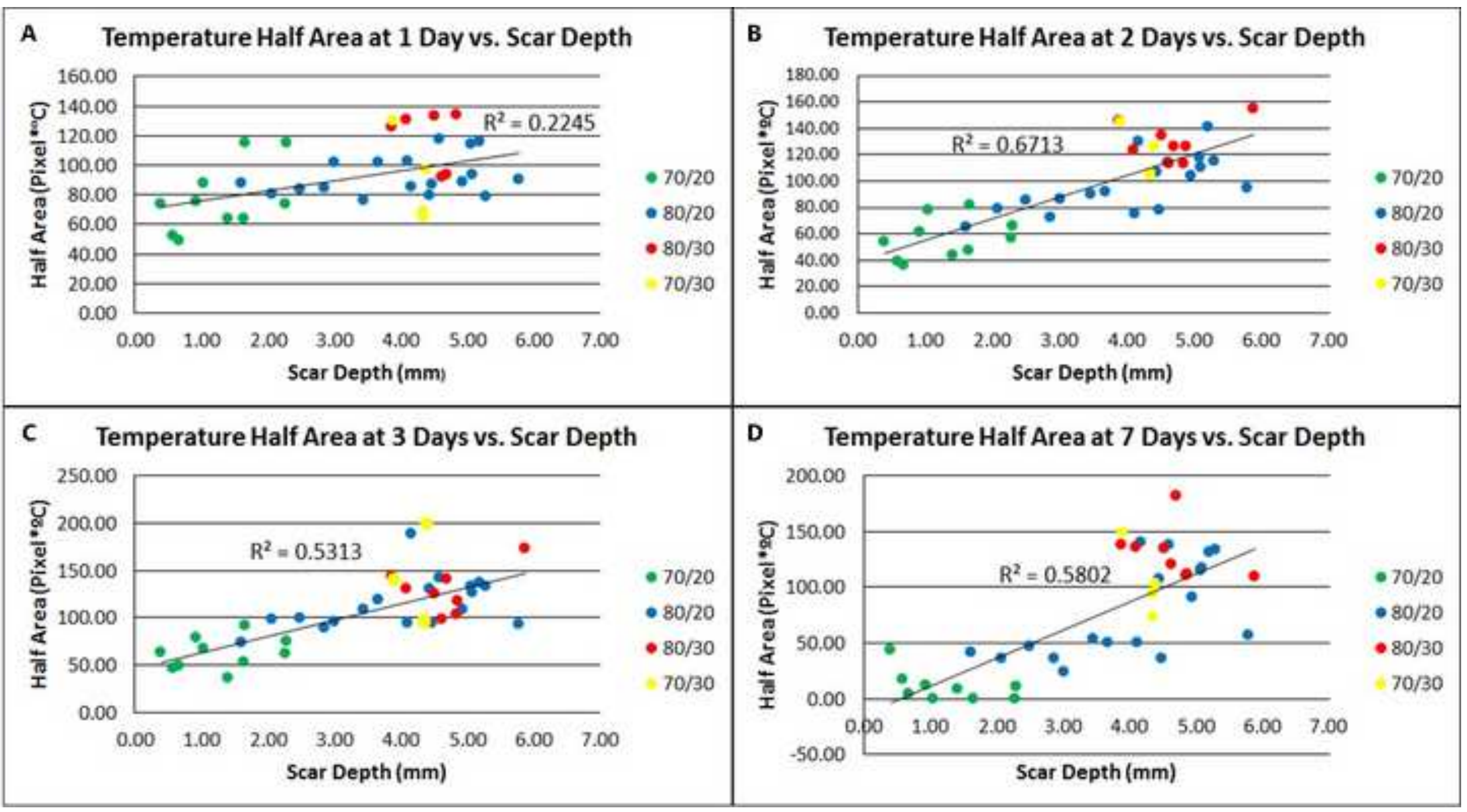



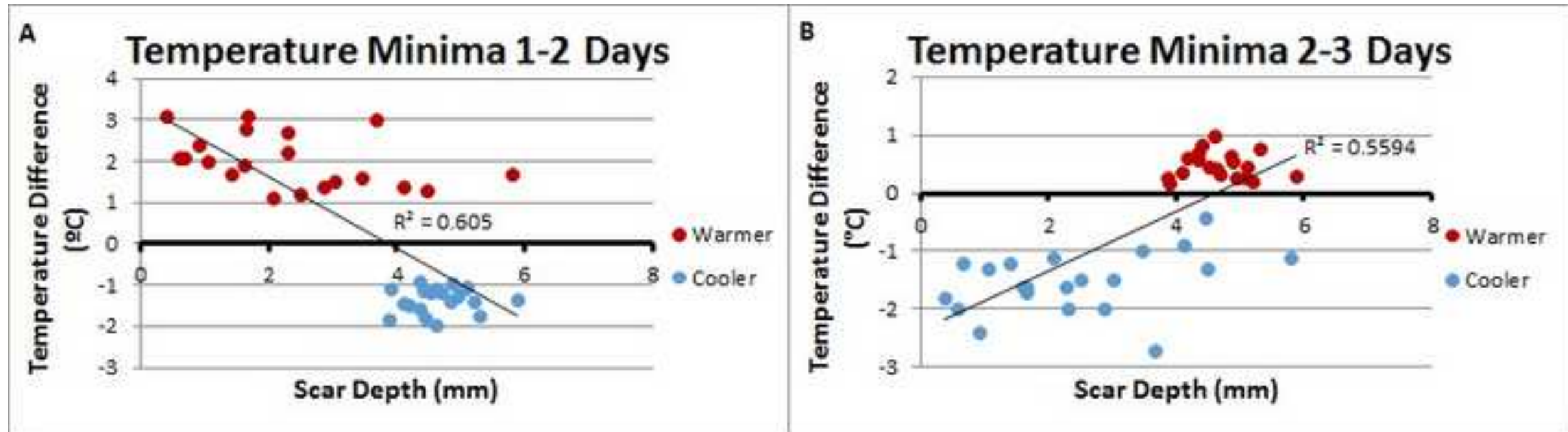

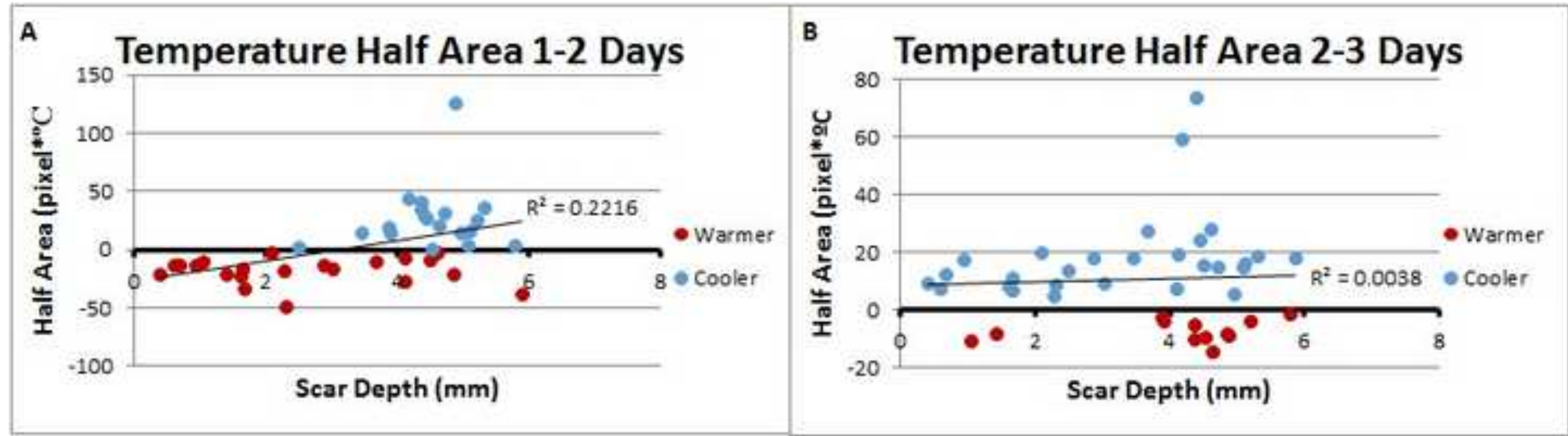
A Diagnostic Test Parameters: Temperature Minima at Day 2

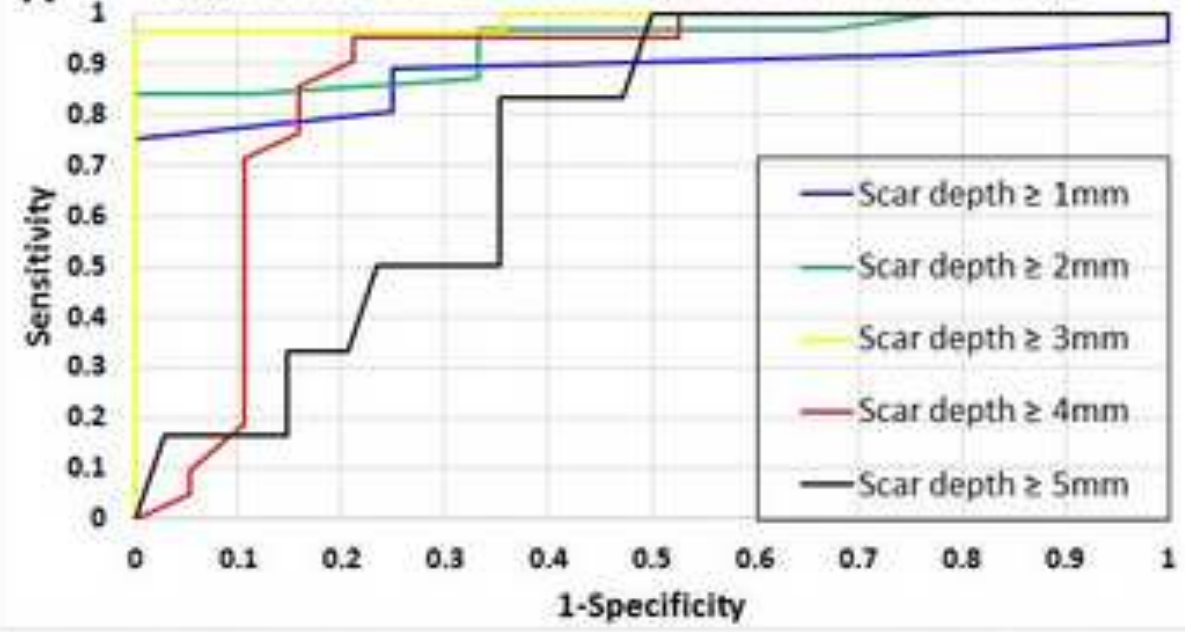

\section{Diagnostic Test Parameters: Temperature half area at Day 2}

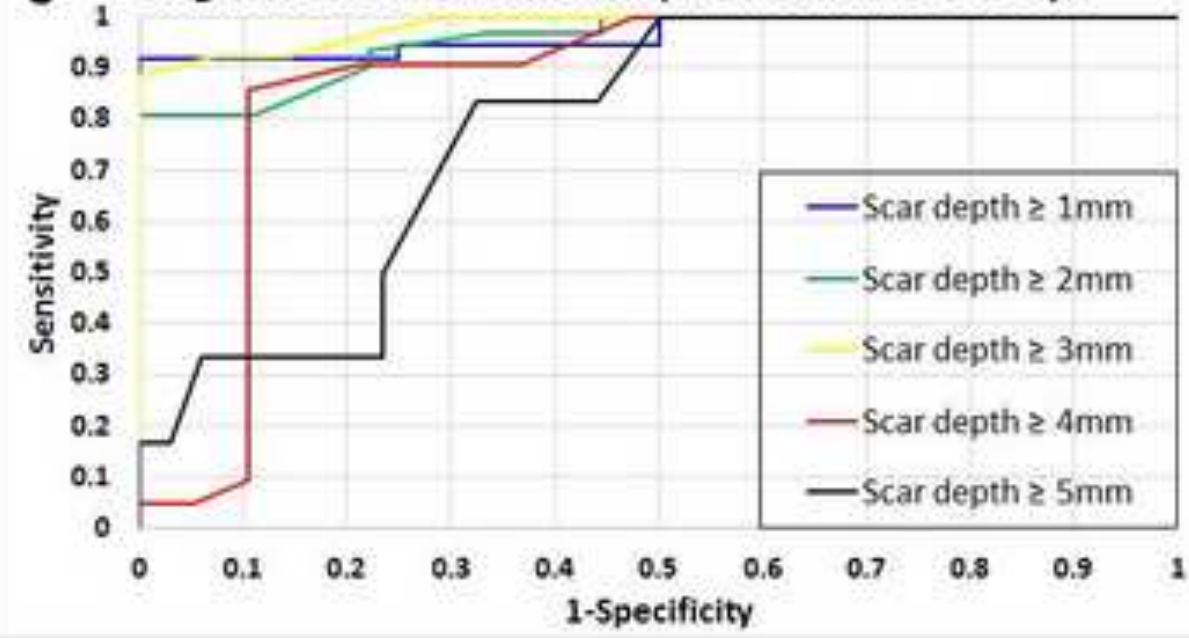

\section{B Dlagnostic Test Parameters: Temperature Minima 1-2 Days}

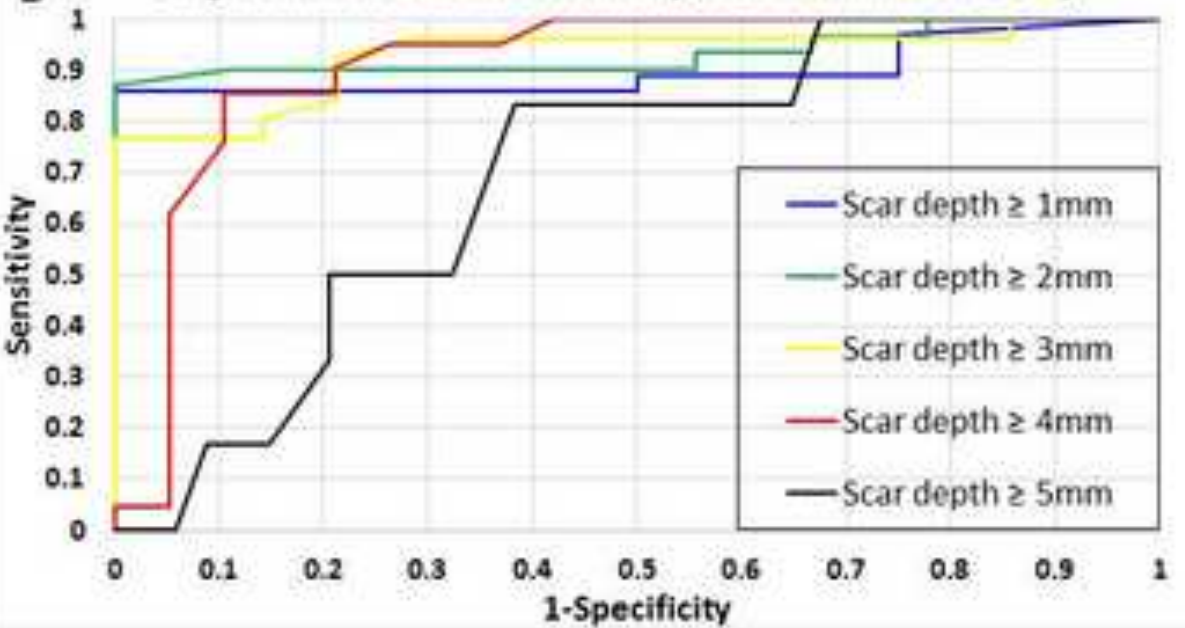

D Diagnostic Test Parameters: Temperature Half Area 1-2 Days

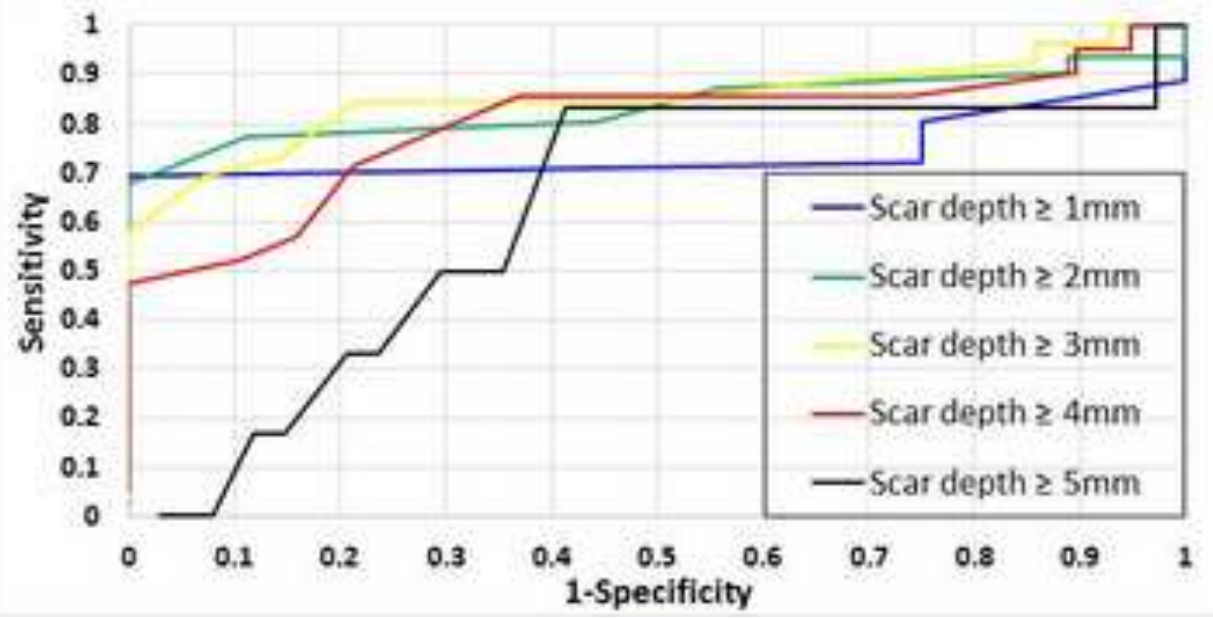


Table 1: Average temperature minima, temperature half area, and scar depth for different burns

\begin{tabular}{|c|c|c|c|c|c|c|c|c|c|}
\hline & \multicolumn{4}{|c|}{ Temperature Minima (Celsius) } & \multicolumn{4}{|c|}{ Temperature Half-Area (Celsius x Pixel) } & \\
\hline Burn Type & 1 day & 2 days & 3 days & 7 days & 1 day & 2 days & 3 days & 7 days & $\begin{array}{l}\text { Scar Depth } \\
(\mathrm{mm})\end{array}$ \\
\hline $70 / 20(n=10)$ & $\begin{array}{c}29.99 \\
\pm 0.63 \\
\end{array}$ & $\begin{array}{c}32.41 \\
\pm 0.35 \\
\end{array}$ & $\begin{array}{c}30.73 \\
\pm 0.52 \\
\end{array}$ & $\begin{array}{c}30.93 \\
\pm 0.47 \\
\end{array}$ & $\begin{array}{l}76.75 \\
\pm 22.97 \\
\end{array}$ & $\begin{array}{l}56.14 \\
\pm 15.45 \\
\end{array}$ & $\begin{array}{r}61.89 \\
\pm 16.84 \\
\end{array}$ & $\begin{array}{l}10.63 \\
\pm 13.86 \\
\end{array}$ & $\begin{array}{r}1.29 \\
\pm 0.68 \\
\end{array}$ \\
\hline $80 / 20(n=18)$ & $\begin{array}{c}30.69 \\
\pm 1.05 \\
\end{array}$ & $\begin{array}{c}30.98 \\
\pm 1.05 \\
\end{array}$ & $\begin{array}{c}30.34 \\
\pm 0.57 \\
\end{array}$ & $\begin{array}{c}29.71 \\
\pm 1.77 \\
\end{array}$ & $\begin{array}{l}92.51 \\
\pm 13.15 \\
\end{array}$ & $\begin{array}{l}97.36 \\
\pm 21.01 \\
\end{array}$ & $\begin{array}{c}114.50 \\
\pm 27.07 \\
\end{array}$ & $\begin{array}{l}77.70 \\
\pm 42.33 \\
\end{array}$ & $\begin{array}{l}4.02 \\
\pm 1.21 \\
\end{array}$ \\
\hline $70 / 30(n=4)$ & $\begin{array}{c}31.00 \\
\pm 1.04 \\
\end{array}$ & $\begin{array}{c}29.83 \\
\pm 0.87 \\
\end{array}$ & $\begin{array}{c}30.40 \\
\pm 1.07 \\
\end{array}$ & $\begin{array}{c}28.45 \\
\pm 1.03 \\
\end{array}$ & $\begin{array}{r}89.81 \\
\pm 30.27 \\
\end{array}$ & $\begin{array}{c}119.99 \\
\pm 19.44 \\
\end{array}$ & $\begin{array}{c}133.43 \\
\pm 48.88 \\
\end{array}$ & $\begin{array}{c}104.43 \\
\pm 32.10 \\
\end{array}$ & $\begin{array}{r}4.25 \\
\pm 0.23 \\
\end{array}$ \\
\hline $80 / 30(n=8)$ & $\begin{array}{c}31.00 \\
\pm 0.71\end{array}$ & $\begin{array}{c}29.47 \\
\pm 0.52\end{array}$ & $\begin{array}{c}29.89 \\
\pm 0.58\end{array}$ & $\begin{array}{c}27.31 \\
\pm 0.37\end{array}$ & $\begin{array}{c}115.46 \\
\pm 20.00\end{array}$ & $\begin{array}{r}129.40 \\
\pm 14.87\end{array}$ & $\begin{array}{r}128.84 \\
\pm 23.71\end{array}$ & $\begin{array}{c}130.37 \\
\pm 24.06\end{array}$ & $\begin{array}{l}4.68 \\
\pm 0.60\end{array}$ \\
\hline
\end{tabular}

Table 2: Changes in Temperature Minima and Temperature Half Area from 1 to 2 days and 2 to 3 days

\begin{tabular}{|c|c|c||c|c|c|}
\hline Delta parameter & Change & $\begin{array}{c}\text { Average } \\
\text { scar depth } \\
\text { (mm) }\end{array}$ & Delta parameter & Change & $\begin{array}{c}\text { Average } \\
\text { scar depth } \\
\text { (mm) }\end{array}$ \\
\hline $\begin{array}{c}\text { Change in } \\
\text { temperature } \\
\text { minima from } \\
1 \text { to 2 days }\end{array}$ & Cooler $(\mathrm{n}=20)$ & $3.88 \pm 0.5$ & $\begin{array}{c}\text { Change in } \\
\text { temperature half } \\
\text { area from } \\
\text { 1 to 2 days }\end{array}$ & Cooler $(\mathrm{n}=19)$ & $4.5 \pm 0.74$ \\
\cline { 2 - 6 } & Warmer $(\mathrm{n}=20)$ & $2.33 \pm 1.43$ & Warmer (n=21) & $2.58 \pm 1.61$ \\
\hline $\begin{array}{c}\text { Change in } \\
\text { temperature } \\
\text { minima from } \\
2 \text { to 3 days }\end{array}$ & Cooler $(\mathrm{n}=21)$ & $2.43 \pm 1.47$ & $\begin{array}{c}\text { Change in } \\
\text { temperature half } \\
\text { area from } \\
\text { 2 to 3 days }\end{array}$ & Cooler $(\mathrm{n}=28)$ & $3.25 \pm 1.62$ \\
\cline { 2 - 6 } & Warmer $(\mathrm{n}=19)$ & $4.68 \pm 0.51$ & Warmer $(\mathrm{n}=12)$ & $4.07 \pm 1.43$ \\
\hline
\end{tabular}

Table 3: Optimal Cutoff Values for Using FLIR to Predict Scar Depth

\begin{tabular}{|c|c|c|c|}
\hline Diagnostic Test & Optimal Cutoff Values & Sensitivity & Specificity \\
\hline Temperature Minima at Day 2 & Scar depth $\geq 3 \mathrm{~mm}, \mathrm{~T} \leq 31.5^{\circ} \mathrm{C}$ & $96 \%$ & $100 \%$ \\
\hline Temperature Minima 1-2 Days & $\begin{array}{c}\text { Scar depth } \geq 2 \mathrm{~mm} \text {, change in temperature } \leq 1.7^{\circ} \mathrm{C} \\
\left.\text { (Scar depth } \geq 3 \mathrm{~mm} \text {, change in temperature } \leq 1.3^{\circ} \mathrm{C}\right)\end{array}$ & $\begin{array}{c}90 \% \\
(81 \%)\end{array}$ & $\begin{array}{c}89 \% \\
(86 \%)\end{array}$ \\
\hline Temperature Half-Area at Day 2 & Scar depth $\geq 3 \mathrm{~mm}$, Half Area $\geq 85$ & $92 \%$ & $93 \%$ \\
\hline Temperature Half-Area 1-2 Days & $\begin{array}{c}\text { Scar depth } \geq 2 \mathrm{~mm} \text {, change in half-area } \geq-10 \\
\text { (Scar depth } \geq 3 \mathrm{~mm} \text {, change in half-area } \geq-10)\end{array}$ & $\begin{array}{c}(85 \%) \\
(79 \%)\end{array}$ \\
\hline
\end{tabular}

